\title{
Magnetic charge, angular momentum and negative cosmological constant
}

\author{
J.J. van der Bij and Eugen Radu| \\ Albert-Ludwigs-Universität \\ Fakultät für Mathematik und Physik, \\ Physikalisches Institut, Freiburg Germany
}

\begin{abstract}
We argue that there are no axially symmetric rotating monopole solutions for a Yang-Mills-Higgs theory in flat spacetime background. We construct axially symmetric Yang-Mills-Higgs solutions in the presence of a negative cosmological constant, carrying magnetic charge $n$ and a nonvanishing electric charge. However, these solution are also nonrotating.
\end{abstract}

\section{Introduction}

Even if nobody will ever see a magnetic monopole, there is surely much to be gained by studying the theory of monopoles. In the seminal paper of Julia and Zee [1] the existence of a profound connection between the angular momentum and the electric charge in a Yang-Mills-Higgs (YMH) theory has been suggested. A well know result in classical electrodynamics implies that in the presence of a magnetic monopole an electrically charged particle acquires an additional angular momentum oriented toward the position of the monopole and with a magnitude equal to the product of the electric and magnetic charges [2]. In the nonabelian case the electric charge distribution and the magnetic charge distribution are not separated. To compute the angular momentum for this type of configuration we have to solve extremely complicated partial differential equations.

Surprisingly, a few years ago it has been shown that Julia-Zee dyons do not admit slowly rotating excitations [3]. However the absence or presence of rotating perturbative solutions, though indicative, is not in general conclusive to establish the absence or presence of exact solutions. For instance in boson star models slowly rotating perturbative solutions are absent, but solutions with a quantized angular momentum exist [4] (see also the discussion in [5]). It is therefore important to identify non-perturbative criteria to settle such questions.

In a recent paper a general relation for the total angular momentum in an Einstein-Yang-Mills-Higgs theory as a surface integral in terms of Yang-Mills (YM) fields has been derived [6]. Within an ansatz which satisfies some extra symmetries it has been found that there are no asymptotically flat rotating dyon solutions, while a monopole-antimonopole configuration with zero net magnetic charge possesses an angular momentum proportional to the electric charge. However Ref.[6] leaves unsolved the question of possible existence of finite energy solutions with a nonvanishing total angular momentum and net magnetic charge for a more general ansatz.

In this paper we argue that, for the most general axially symmetric YMH monopole configuration, the total angular momentum is zero. We adress also the same question for a different asymptotic structure of spacetime and present arguments for the existence of axially symmetric dyon solutions in an anti-de Sitter (AdS) spacetime. Qualitatively, the behavior of AdS solutions is very similar to that corresponding to

\footnotetext{
${ }^{1}$ corresponding author:

Albert-Ludwigs-Universität Freiburg,

Fakultät für Mathematik und Physik, Physikalisches Institut,

Hermann-Herder-Straße 3, D-79104 Freiburg, Germany

email: radu@newton.physik.uni-freiburg.de

Telephone: +49-761/203-7630

Fax: $+49-761 / 203-5967$
} 
Minkowski spacetime configurations. However, we argue that these solutions present also a vanishing total angular momentum.

\section{General ansatz and total angular momentum}

To simplify the general picture, in this paper we ignore the effects of gravity. We follow also the notations and conventions used by Hartmann, Kleihaus and Kunz in [7]. The action for a non-Abelian $S U(2)$ gauge field coupled to a triplet Higgs field with the usual potential $V(\Phi)=\frac{\lambda}{8} \operatorname{Tr}\left(\Phi^{2}-\eta^{2}\right)^{2}$ is

$$
S=\int d^{4} x \sqrt{-g}\left[\operatorname{Tr}\left\{\frac{1}{2} F_{\mu \nu} F^{\mu \nu}\right\}+\operatorname{Tr}\left\{\frac{1}{2} D_{\mu} \Phi D^{\mu} \Phi\right\}+V(\Phi)\right],
$$

where the field strength tensor is

$$
F_{\mu \nu}=\partial_{\mu} A_{\nu}-\partial_{\nu} A_{\mu}+i\left[A_{\mu}, A_{\nu}\right]
$$

and the covariant derivative

$$
D_{\mu}=\partial_{\mu}+i\left[A_{\mu},\right]
$$

Varying the action (1) with respect to $A_{\mu}$ and $\Phi$ we have the field equations

$$
\begin{aligned}
\frac{1}{\sqrt{-g}} D_{\mu}\left(\sqrt{-g} F^{\mu \nu}\right) & =\frac{1}{4} i\left[\Phi, D^{\nu} \Phi\right], \\
\frac{1}{\sqrt{-g}} D_{\mu}\left(\sqrt{-g} D^{\mu} \Phi\right)+\lambda\left(\Phi^{2}-\eta^{2}\right) \Phi & =0 .
\end{aligned}
$$

Varying the Lagrangian with respect to the metric $g_{\mu \nu}$ yields the energy-momentum tensor

$$
T_{\mu \nu}=2 \operatorname{Tr}\left\{F_{\mu \alpha} F_{\nu \beta} g^{\alpha \beta}-\frac{1}{4} g_{\mu \nu} F_{\alpha \beta} F^{\alpha \beta}\right\}+\operatorname{Tr}\left\{\frac{1}{2} D_{\mu} \Phi D_{\nu} \Phi-\frac{1}{4} g_{\mu \nu}\left(D_{\alpha} \Phi D^{\alpha} \Phi\right\}+V(\Phi) g_{\mu \nu} .\right.
$$

The energy density of a solution of the YMH equations is given by the $t t$-component of the energymomentum tensor. Integration over all space yields the total energy

$$
\begin{aligned}
E & =-\int T_{t}^{t} \sqrt{-g} d^{3} x=\int_{0}^{\infty} d r \int_{0}^{\pi} d \theta \int_{0}^{2 \pi} d \varphi \sqrt{-g} \operatorname{Tr}\left\{F_{r \theta} F^{r \theta}+F_{r \varphi} F^{r \varphi}+F_{\theta \varphi} F^{\theta \varphi}\right. \\
& \left.-F_{r t} F^{r t}-F_{\theta t} F^{\theta t}-F_{\varphi t} F^{\varphi t}+\frac{1}{4}\left(D_{r} \Phi D^{r} \Phi+D_{\theta} \Phi D^{\theta} \Phi+D_{\varphi} \Phi D^{\varphi} \Phi-D_{t} \Phi D^{t} \Phi\right)+\frac{\lambda}{8}\left(\Phi^{2}-\eta^{2}\right)^{2}\right\},
\end{aligned}
$$

where $r, \theta, \varphi$ are the usual spherical coordinates. For the energy integral to converge, each of the terms in (5) must vanish at large $r$. The finite energy condition imposed on this expression will give us the acceptable asymptotic behavior of the gauge functions and Higgs field as $r \rightarrow \infty$. The additional requirements to have a finite, locally integrable energy density impose boundary conditions at the origin and on the $z$-axis.

The total angular momentum is

$$
J=\int T_{\varphi}^{t} \sqrt{-g} d^{3} x=\int 2 \operatorname{Tr}\left\{F_{r \varphi} F^{r t}+F_{\theta \varphi} F^{\theta t}+D_{\varphi} \Phi D^{t} \Phi\right\} \sqrt{-g} d^{3} x .
$$

We mention also the expression for the electric and magnetic charges derived by using the 't Hooft field strength tensor

$$
\begin{aligned}
Q_{e} & =\frac{1}{4 \pi} \oint_{\infty} d S_{\mu} \operatorname{Tr}\left\{\hat{\Phi} F_{\mu t}\right\} \\
Q_{m} & =\frac{1}{4 \pi} \oint_{\infty} d S_{\mu} \frac{1}{2} \epsilon_{\mu \nu \alpha} \operatorname{Tr}\left\{\hat{\Phi} F_{\nu \alpha}\right\}
\end{aligned}
$$


where the integration is over a surface at spatial infinity and $\hat{\Phi}=\Phi /|\Phi|$.

We are interested in static, axially symmetric finite energy solutions of the equations (2)-(32). As proven in [6], for static axially symmetric configurations, the volume integral (6) can be converted into a surface integral in terms of Yang-Mills potentials. The symmetry of the gauge field under a spacetime symmetry means that the action of an isometry can be compensated by a suitable gauge transformation [8, 9]. For the time translational symmetry, we choose a natural gauge such that $\partial A / \partial t=0$. However, a rotation around the $z$-axis can be compensated by a gauge rotation

$$
\mathcal{L}_{\varphi} A=D \Psi
$$

and therefore

$$
\begin{aligned}
F_{\mu \varphi} & =D_{\mu} W \\
D_{\varphi} \Phi & =i[W, \Phi]
\end{aligned}
$$

where $W=A_{\varphi}-\Psi$.

Using the potential $W$ we find

$$
\begin{aligned}
T_{\varphi}^{t}= & 2 \operatorname{Tr}\left\{\left(D_{r} W\right) F^{r t}+\left(D_{\theta} W\right) F^{\theta t}+\frac{i}{4}[W, \Phi] D^{t} \Phi\right\} \\
= & 2 \operatorname{Tr}\left\{\frac{1}{\sqrt{-g}} D_{r}\left(W F^{r t} \sqrt{-g}\right)+\frac{1}{\sqrt{-g}} D_{\theta}\left(W F^{\theta t} \sqrt{-g}\right)\right. \\
& \left.-W\left(\frac{1}{\sqrt{-g}} D_{r}\left(\sqrt{-g} F^{r t}\right)+\frac{1}{\sqrt{-g}} D_{\theta}\left(\sqrt{-g} F^{\theta t}\right)\right)+\frac{i}{4}[W, \Phi] D^{t} \Phi\right\} .
\end{aligned}
$$

As a consequence of the YM equations (2) and making use of the fact that the trace of a commutator vanishes we obtain

$$
T_{\varphi}^{t}=2 \operatorname{Tr}\left\{\frac{1}{\sqrt{-g}} \partial_{\mu}\left(W F^{\mu t} \sqrt{-g}\right)\right\} .
$$

Thus, ignoring possible singularities the expression of the total angular momentum is

$$
\begin{aligned}
J & =\oint_{\infty} 2 \operatorname{Tr}\left\{W F^{\mu t}\right\} d S_{\mu} \\
& =2 \pi \lim _{r \rightarrow \infty} \int_{0}^{\pi} d \theta \sin \theta r^{2}\left[W^{(r)} F^{r t(r)}+W^{(\theta)} F^{r t(\theta)}+W^{(\varphi)} F^{r t(\varphi)}\right] .
\end{aligned}
$$

Note that this relation has been derived without fixing the gauge. It greatly simplifies the evaluation of the angular momentum of a regular YMH configuration, since we need only the asymptotics of some gauge functions. In what follows, we use (13) to prove that the total angular momentum of a finite energy dyon solution in flat space is zero.

The method of proof is, in principle, quite straightforward. From the assumptions of finite energy density and finite total energy, it follows that every term in the energy integral (5) must be finite. The most crucial requirement is that $D_{\mu} \Phi$ vanish sufficiently rapidly as $r \rightarrow \infty$. When evaluating (13) we find a vanishing $J$ for this behavior.

The construction of an axially symmetric YMH ansatz has been discussed by many authors starting with the pioneering papers by Manton [10] and Rebbi and Rossi [11]. The most general axially symmetric Yang-Mills ansatz contains 12 functions: 9 magnetic and 3 electric potentials and can be easily obtained in cylindrical coordinates $x^{\mu}=(\rho, \varphi, z, t)$ (with $\left.\rho=r \sin \theta, z=r \cos \theta\right)$

$$
A_{\mu}=\frac{1}{2} A_{\mu}^{\rho}(\rho, z) \tau_{\rho}^{n}+\frac{1}{2} A_{\mu}^{\varphi}(\rho, z) \tau_{\varphi}^{n}+\frac{1}{2} A_{\mu}^{z}(\rho, z) \tau_{z}
$$


where the only $\varphi$-dependent terms are the $S U(2)$ matrices (composed of the standard $\left(\tau_{x}, \tau_{y}, \tau_{z}\right)$ Pauli matrices)

$$
\begin{aligned}
& \tau_{\rho}^{n}=\cos n \varphi \tau_{x}+\sin n \varphi \tau_{y}, \\
& \tau_{\varphi}^{n}=-\sin n \varphi \tau_{x}+\cos n \varphi \tau_{y} .
\end{aligned}
$$

We can reduce this general ansatz by imposing some extra symmetries on the fields. To our knowledge there are no analytical or numerical results for the most general ansatz.

Transforming to spherical coordinates, it is convenient to introduce, without any loss of generality, a new $S U(2)$ basis $\left(\tau_{r}^{n}, \tau_{\theta}^{n}, \tau_{\varphi}^{n}\right)$, with

$$
\begin{aligned}
\tau_{r}^{n} & =\sin \theta \tau_{\rho}^{n}+\cos \theta \tau_{z}, \\
\tau_{\theta}^{n} & =\cos \theta \tau_{\rho}^{n}-\sin \theta \tau_{z} .
\end{aligned}
$$

Thus, similar to (14), we find the general expression

$$
A_{\mu}=\frac{1}{2} A_{\mu}^{r}(r, \theta) \tau_{r}^{n}+\frac{1}{2} A_{\mu}^{\theta}(r, \theta) \tau_{\theta}^{n}+\frac{1}{2} A_{\mu}^{\varphi}(r, \theta) \tau_{\varphi}^{n},
$$

where $A_{\mu}^{a} d x^{\mu}=A_{r}^{a} d r+A_{\theta}^{a} d \theta+A_{\varphi}^{a} d \varphi+A_{t}^{a} d t$. For the Higgs field we have a similar relation $\Phi=\Phi^{a}(r, \theta) \tau_{a}^{n}$. The gauge invariant quantities expressed in terms of these functions will be independent of the azimutal angle $\varphi$ and hence axially symmetric. The winding number $n$ corresponds to the topological charge of the solutions and is a constant of motion.

For this parametrization

$$
\Psi=n \frac{\tau_{z}}{2}=n \cos \theta \frac{\tau_{r}^{n}}{2}-n \sin \theta \frac{\tau_{\theta}^{n}}{2} .
$$

The Higgs field must be nonvanishing at spatial infinity in order that the potential energy be zero there. Thus, the spontaneous symmetry-breaking mechanism requires

$$
\lim _{r \rightarrow \infty} \Phi^{a} \Phi^{a}=\eta^{2} .
$$

As proven in [12], any regular axially symmetric magnetic charge distribution can be located only at isolated points situated on the axis of symmetry, with equal and opposite values of the charge at alternate points. In particular, if only one sign of the charge is allowed, all the charge must be concentrated at a single point.

For a multimonopole solution containing only magnetic charges with the same sign, the suitable boundary conditions for the Higgs field at infinity are $\Phi^{r}=\eta, \Phi^{\theta}=\Phi^{\varphi}=0$. Note that a more general asymptotic behavior of the Higgs field is allowed by (19), but this would correspond to composed configurations containing both monopoles and antimonopoles.

The requirement of finite total energy implies the decay of the $F_{r t}^{a}$ as $r \rightarrow \infty$ is faster than $1 / r^{1.5}$. Also, the contribution $\left(D_{\varphi} \Phi\right)^{a}\left(D_{\varphi} \Phi\right)^{a} g^{\varphi \varphi}$ term to the energy is finite if $\left(D_{\varphi} \Phi\right)^{a}$ approaches zero to large $r$ sufficiently rapid. This implies the fall-off conditions $\left(W_{\theta}, W_{\varphi}\right) \sim 1 / r^{0.5+\epsilon}$ for large $r$ (with $\epsilon>0$ ). Therefore the last two terms in (13) give null contribution to the total angular momentum. The contribution of the $A_{\varphi}^{r}$ to the total angular momentum is also zero, since it vanishes asymptotically for a regular configuration.

By using these results we find

$$
\lim _{r \rightarrow \infty} \operatorname{Tr}\left(r^{2} W F_{r t}\right)=-\frac{n Q_{e}}{2} \cos \theta
$$

where $Q_{e}$ is the electric charge of the configuration given by (7). Therefore the total angular momentum of the solution is clearly zero. Thus, an axially symmetric regular dyon has a vanishing total angular momentum. 


\section{Axially symmetric dyons in AdS background}

This nonexistence result can be partially attributed to the asymptotic structure of spacetime. One may ask whether it remains valid in the presence of a cosmological constant $\Lambda$.

However, up to date, all known axially symmetric dyon regular solutions have been obtained in a fixed Minkowski background. In what follows we consider dyon solutions in an AdS spacetime. Further motivation for this attempt comes from the surprising behavior found for a $S U(2)$-gauge field in the presence of a negative cosmological constant. The existence of stable particle-like and black hole solutions in an EinsteinYang-Mills theory with $\Lambda<0$ is the most exciting result [13, 14].

The global existence of a solution of the Cauchy problem for the YMH equations in AdS spacetime is considered in 15. Some analytical properties of spherically symmetric monopole and dyon solutions for YMH theory with a negative cosmological constant have been discussed in [16]. Unlike the flat spacetime case, in an AdS spacetime there are no analytic solutions that might be used as a guiding line. Moreover, when a cosmological constant is included (no matter how small this constant is) no solution close to the BPS configuration can be found. As proven in [16], it is the change in the asymptotic behavior of the Higgs field that prevents such a solution. Numerical monopole solutions have been exhibited in [17], where the effects of gravity are also included. A distinctive feature of AdS solutions concerns the asymptotic behavior of the fields. When $\Lambda<0$, the Higgs field approaches its vacuum expectation value faster than in the flat space case. The radius of the monopole core decreases, as the magnetic field concentrates near the origin.

In what follows, we present numerical arguments for the existence of spherically- and axially symmetric dyon solutions in an AdS spacetime with line-element

$$
d s^{2}=\frac{d r^{2}}{1-\frac{\Lambda}{3} r^{2}}+r^{2}\left(d \theta^{2}+r^{2} \sin ^{2} \theta d \varphi^{2}\right)-\left(1-\frac{\Lambda}{3} r^{2}\right) d t^{2} .
$$

Searching for general axially symmetric solutions within the most general ansatz is a difficult task. In our approach we consider a reduced ansatz used also to obtain flat space - axially symmetric dyons 18

$$
\begin{aligned}
& A_{r}^{r}=A_{r}^{\theta}=A_{\theta}^{r}=A_{\theta}^{\theta}=A_{\varphi}^{\varphi}=\Phi^{\varphi}=0, \\
& A_{r}^{r}=\frac{H_{1}}{r}, A_{\theta}^{r}=1-H_{2}, A_{\varphi}^{r}=-n \sin \theta H_{3}, A_{\varphi}^{\theta}=-n \sin \theta\left(1-H_{4}\right), \\
& A_{t}^{r}=\eta H_{5}, A_{t}^{\theta}=\eta H_{6}, \Phi^{r}=\eta \Phi_{1}, \Phi^{\theta}=\eta \Phi_{2} .
\end{aligned}
$$

This ansatz satisfies some discrete symmetries [19] and was used by various authors when discussing numerically solutions [7]. The boundary conditions at infinity consistent with the requirements of regularity, finite energy and symmetry, are

$$
H_{i}=0, i=1,2,3,4,6 ; \quad H_{5}=\alpha ; \quad \Phi_{1}=1 ; \quad \Phi_{2}=0
$$

at infinity, and

$$
H_{i}=0, i=1,3,5,6 ; \quad H_{2}=H_{4}=1, \quad \Phi_{1}=\Phi_{2}=0,
$$

at the origin. Given the parity reflection symmetry, we need to consider solutions only in the region $0 \leq \theta \leq$ $\pi / 2$; on the $z$ - and $\rho$-axis the functions $H_{1}, H_{3}, H_{6}, \Phi_{2}$ and the derivatives $\partial_{\theta} H_{2}, \partial_{\theta} H_{4}, \partial_{\theta} H_{5}$ and $\partial_{\theta} \Phi_{1}$ are to vanish. To fix the residual abelian gauge invariance we choose the usual gauge condition $r \partial_{r} H_{1}-\partial_{\theta} H_{2}=0$ [7. 18 .

Within this ansatz, we have found dyon solutions for any value of the cosmological constant. We change to dimensionless coordinates and Higgs field by rescaling $r \rightarrow r / \eta, \Lambda \rightarrow \Lambda \eta^{2}$ and $\Phi \rightarrow \eta \Phi$, respectively. The numerical calculations for $n>1$ were performed with the software package CADSOL/FIDISOL, based on the Newton-Raphson method [20]. We use the general relations (5)-(8) to compute the energy, angular momentum and charges of these configurations. The solutions with $n=1$ corresponds to spherically symmetric dyons with $H_{1}=H_{3}=\Phi_{2}=0, H_{2}=H_{4}=w(r), \Phi_{1}=H(r), H_{5}=J(r)$, and generalize the Julia-Zee solution [1] for a negative cosmological constant. Typical spherically symmetric solutions are presented in Fig. 1. 
Although the results we find are broadly similar to those valid for the $\Lambda=0$ case, there are some differences. The most interesting difference, not discussed in [16], is that the magnitude of the electric potential $A_{t}$ at infinity is no longer restricted. In an asymptotically Minkowski spacetime, the constant $\alpha$ in eq. (23) is restricted to $\alpha \leq 1$. For $\alpha>1$ some gauge field functions become oscillating instead of asymptotically decaying. The $A_{t}^{a}$ components of the gauge filed act like an isotriplet Higgs field with negative metric, and by themselves would cause the other components of the gauge field to oscillate rather than decrease exponentially as $r \rightarrow \infty$ [1. 18]. However, in a AdS spacetime, finite energy solutions with arbitrary $\alpha$ are allowed. The Higgs field forces $H_{1}=H_{2}=H_{4}=H_{6}=0$ asymptotically, but does not restrict the value of $H_{5}$. In the limit of vanishing Higgs field $(\eta \rightarrow 0)$ we recover the (pure-) YM solutions discussed in 21, 22 (in the absence of the Higgs field the value at infinity of the magnetic potentials $H_{2}, H_{4}$ is arbitrary). Another distinctive feature of the AdS solutions is a smaller dyon radius as compared to the $\Lambda=0$ case.

Apart from these properties, qualitatively, the Higgs field and Yang-Mills field behavior is very similar to that corresponding to Minkowski spacetime dyons. We notice a similar shape for the functions $H_{i}$ and $\Phi_{i}$ and also for the energy density. Increasing in size at the origin for increasing $|\Lambda|$, the energy density is localized in a decreasing region of space. The energy of the multidyons is on order of $n$ times the corresponding one-dyon energy. In Fig. 2 the energy and the electric charge of the solutions is plotted as a function of the cosmological constant for various winding numbers. The toroidal shape familiar from the energy density of the dyon solutions in flat space is retained for solutions in AdS space, as is illustrated in Fig. 3.

Most of our results have been obtained in the Prasad-Sommerfeld limit, but similar conclusions hold for a nonvanishing Higgs self-coupling. Further details on these solutions will be presented elsewhere.

Returning to the question of angular momentum, by using the ansatz (22) with the boundary conditions (23) we find again

$$
J=-2 \pi \lim _{r \rightarrow \infty} \int_{0}^{\pi} d \theta \sin \theta r^{2} W^{(r)} F_{r t}^{(r)}=2 \pi n Q_{e} \int_{0}^{\pi} d \theta \sin \theta \cos \theta=0 .
$$

It is worthwhile to notice that these solutions carry a nonvanishing angular momentum density, even in the Prasad-Sommerfeld limit, as ilustrated in Fig. 3c (see also Fig. 4). The fact that $J=0$ can be atributed again to the extra symmetries of the reduced ansatz, since as $\theta \rightarrow \pi-\theta$ we have $H_{1} \rightarrow-H_{1}, H_{2} \rightarrow$ $H_{2}, H_{3} \rightarrow-H_{3}, H_{4} \rightarrow H_{4}, H_{5} \rightarrow H_{5}, H_{6} \rightarrow-H_{6}$ which implies the vanishing of the integral (6). We find also that the YM dyon solutions discussed in 21] have a vanishing angular momentum.

Evidently, dyon solutions within the most general ansatz may also exist. The construction of such solutions represents a difficult challenge. However, we argue that even for the most general ansatz, an AdS finite energy dyon solution presents a vanishing total angular momentum.

The asymptotic behavior of the functions $A_{\mu}^{a}, \Phi^{a}$ is complicated by the presence of a negative cosmological constant. Similar to the $n=1$ case [16, 17], the transversal components of the YM potentials present a complicated power decay, instead of the expected exponential law. Nevertheless, following the analysis in the $\Lambda=0$ case, we find that the required fall-off conditions for a finite total energy are again $F_{r t}^{a} \sim 1 / r^{1.5+\epsilon}$, $W_{\theta}$ and $W_{\varphi} \sim 1 / r^{0.5+\epsilon}$, while $A_{\varphi}^{r}$ shold also vanish for a regular configuration. Thus, after replacing in (12) we find again $J=0$.

\section{Further discussion}

We presented arguments that the results obtained within a perturbative approach in [3] remain valid in the general case, for the most general YMH axially symmetric ansatz. A flat space axially symmetric dyon configuration necessarily possesses a vanishing total angular momentum.

We emphasize the role played by the Higgs field boundary condition at infinity in obtaining this nonexistence result. We can of course consider a different behavior at infinity, consistent also with (19) and finite energy conditions, corresponding, however, to composed configurations. A sufficiently general asymptotic behavior of the Higgs field at infinity will read

$$
\Phi^{r}=\eta \cos f(\theta), \quad \Phi^{\theta}=\eta \sin f(\theta), \quad \Phi^{\varphi}=0,
$$


where $f(\theta)$ is an arbitrary function of $\theta$ reflecting a gauge freedom. The regularity along the $z$-axis imposes that $\sin f(0)=\sin f(\pi)=0$. Therefore we find two possible sets of boundary conditions: $\cos f(0)=$ $\cos f(\pi)= \pm 1$ or $\cos f(0)=-\cos f(\pi)= \pm 1$ (see also the discussion in [23]). We call the first set even boundary conditions, while the second set (for $f(\pi)=f(0)+(2 k+1) \pi$ ) will be refered to as odd boundary conditions.

For the generic Higgs field boundary conditions (26) and within the reduced ansatz (22), we find the asymptotic behavior in a flat space background

$$
\begin{aligned}
H_{1} & =0, \quad H_{2}=-f^{\prime}(\theta), \quad H_{5}=\alpha \cos f(\theta), H_{6}=\alpha \sin f(\theta), \\
F_{r t}^{(r)} & =\frac{Q_{e} \cos f(\theta)}{r^{2}}, \quad F_{r t}^{(\theta)}=\frac{Q_{e} \sin f(\theta)}{r^{2}} .
\end{aligned}
$$

For even boundary conditions, the regularity and finite energy conditions impose the asymptotic expression of the gauge potentials $\mathrm{H}_{3}, \mathrm{H}_{4}$

$$
H_{3}=\frac{\cos \theta \cos f(0)}{\sin \theta}(\cos f(\theta)-\cos f(0)), \quad H_{4}=-\frac{\cos \theta}{\sin \theta} \sin f(\theta) \cos f(0),
$$

while for the second type of boundary conditions we find

$$
H_{3}=\frac{\cos f(0)}{\sin \theta}(\cos f(\theta)-\cos \theta \cos f(0)), \quad H_{4}=-\frac{\cos f(0) \sin f(\theta)}{\sin \theta} .
$$

By using these expressions and the relations (8), (13) we find that the even boundary configurations have a magnetic charge $Q_{m}= \pm n$ and a vanishing total angular momentum. The configurations satisfying oddboundary conditions present a complementary picture: they posses a zero net magnetic charge and a nonzero angular momentum proportional to the electric charge $J= \pm 4 \pi n Q_{e}$. Thus, as conjectured in [6], it seems that the vanishing of the total angular momentum is related to the presence of a net magnetic charge.

At this stage, we remark that the ansatz (22) is invariant under a gauge transformation $U=$ $\exp \left\{i \Gamma(r, \theta) \tau_{\varphi}^{n} / 2\right\}$ [7], and the Higgs field transforms as

$$
\begin{aligned}
& \Phi^{r} \rightarrow \quad \cos \Gamma \Phi^{r}+\sin \Gamma \Phi^{\theta}, \\
& \Phi^{\theta} \rightarrow-\sin \Gamma \Phi^{r}+\cos \Gamma \Phi^{\theta},
\end{aligned}
$$

which implies $f \rightarrow f-\Gamma(\theta)$ in $(26)$. We may use this relation to set $f(\theta)=k \theta$ in the Higgs field boundary conditions (with $k$ an integer number).

A number of well-known configurations (in a flat space background) are obtained for this choice of $f$. The dyon configurations discussed above correspond to $k=0$. For $k=1$, we have as $r \rightarrow \infty$

$$
\Phi^{r}=\eta \cos \theta, \quad \Phi^{\theta}=\eta \sin \theta, \quad \Phi^{\varphi}=0,
$$

which implies the asymptotic behavior, within the reduced ansatz (22)

$$
\begin{aligned}
H_{1} & =H_{3}=0, \quad H_{2}=H_{4}=-1, \quad H_{5}=\alpha \cos \theta, \quad H_{6}=\alpha \sin \theta, \\
W^{(r)} & =-n \cos \theta, \quad W^{(\theta)}=-n \sin \theta, \quad W^{(\varphi)}=0, \\
F_{r t}^{(r)} & =\frac{Q_{e} \cos \theta}{r^{2}}, \quad F_{r t}^{(\theta)}=\frac{Q_{e} \sin \theta}{r^{2}} .
\end{aligned}
$$

This corresponds to a monopole-antimonopole configuration, with a vanishing net magnetic charge but a nonzero electric charge. This regular solution is discussed in [24, 25] for $n=1, H_{5}=H_{6}=0$ and a different basis $\tau_{a}^{n}$ in spherical coordinates. The authors of these papers use a different combination of $\tau_{\rho}^{n}$ and $\tau_{z}$, more suitable for these boundary conditions $\left(\tau_{r}^{n}=\sin 2 \theta \tau_{\rho}^{n}+\cos 2 \theta \tau_{z}, \tau_{\theta}^{n}=\cos 2 \theta \tau_{\rho}^{n}-\sin 2 \theta \tau_{z}\right)$. In [6] it was found that the total angular momentum of this solution is proportional to the electric charge.

For $k=2$ we find a configuration consisting in two monopoles and one antimonopole (we consider only the case $n=1$ ). This configuration is sustained by the boundary conditions at infinity

$$
\Phi^{r}=\eta \cos 2 \theta, \quad \Phi^{\theta}=\eta \sin 2 \theta, \quad \Phi^{\varphi}=0,
$$


which imply asymptotically

$$
\begin{aligned}
W^{(r)} & =-\cos \theta \cos 2 \theta, \quad W^{(\theta)}=-\cos \theta \sin 2 \theta, \quad W^{(\varphi)}=0, \\
F_{r t}^{(r)} & =\frac{Q_{e} \cos 2 \theta}{r^{2}}, \quad F_{r t}^{(\theta)}=\frac{Q_{e} \sin 2 \theta}{r^{2}},
\end{aligned}
$$

(corresponding to $H_{1}=0, H_{2}=-2, H_{3}=-\sin 2 \theta, H_{4}=-\cos 2 \theta-1, H_{5}=\alpha \cos 2 \theta, H_{6}=\alpha \sin 2 \theta$ for the ansatz (22)). One can verify that this configuration has a unit magnetic charge and a zero total angular momentum.

One may speculate on the existence of solutions for an arbitrary integer $k$, representing composed configurations, containing both monopoles and anti-monopoles. It appears that further analysis of such problems will require at least some use of numerical techniques. The role played by the Higgs field topology and the extra-symmetries of the ansatz needs further study also.

The inclusion of a negative cosmological constant in a YMH theory leads to the same general picture. Numerical results have been presented showing the existence of dyon solutions in fixed AdS spacetime. However, similar to the flat space counterparts, these dyon solutions present also a vanishing total angular momentum. We can consider of course more general configurations containing both monopoles and antimonopoles, by using the set of boundary conditions at infinity derived above. In this case, it is possible to write a similar relation between the electric charge and the angular momentum.

We expect that the inclusion of gravity in this analysis will not change these conclusions, since the gravitational field does not affect the behavior at infinity of the YMH system.

Although the obtained results are in agreement with the perturbative approach, we still are confronted by the problem of the physical mechanism preventing a configuration with net magnetic charge from rotating. A more general proof, beyond a specific ansatz (possibly based on topological arguments), is clearly necessary.

\section{Acknowledgement}

One of the authors (E.R.) acknowledges an important discussion with Erick Wöhnert.

This work was performed in the context of the Graduiertenkolleg of the Deutsche Forschungsgemeinschaft (DFG): Nichtlineare Differentialgleichungen: Modellierung,Theorie, Numerik, Visualisierung.

\section{References}

[1] B. Julia, A. Zee, Phys.Rev.D11 (1975) 2227.

[2] J. D. Jackson, Classical Electrodynamics, Willey\&Sons, New York, 1998.

[3] M. Heusler, N. Straumann, M. S. Volkov, Phys.Rev.D58 (1998) 105021.

[4] S. Yoshida, Y. Eriguchi, Phys.Rev.D56 (1997) 762;

F.E. Schunck and E.W. Mielke, Rotating boson stars, in: Relativity and Scientific Computing, edited by F.W. Hehl, R.A. Puntigam and H. Ruder, Springer (1996) 138-151.

[5] M. S. Volkov and E. Wohnert, arXiv:hep-th/0205157.

[6] J. J. van der Bij and E. Radu, Int. J. Mod. Phys. A 17 (2002) 1477.

[7] B. Hartmann, B. Kleihaus and J. Kunz, Phys. Rev. D 65 (2002) 024027.

[8] M. Heusler, Helv. Phys. Acta 69 (1996) 501.

[9] P. Forgacs and N. S. Manton, Commun. Math. Phys. 72 (1980) 15, P. G. Bergmann and E. J. Flaherty, J. Math. Phys. 19 (1978) 212.

[10] N. S. Manton, Nucl. Phys. B 135 (1978) 319. 
[11] C. Rebbi and P. Rossi, Phys. Rev. D 22 (1980) 2010.

[12] P. Houston and L. O'Raifeartaigh, Z. Phys. C 8 (1981) 175.

[13] E. Winstanley, Class. Quant. Grav. 16 (1999) 1963.

[14] J. Bjoraker and Y. Hosotani, Phys. Rev. D 62 (2000) 043513.

[15] Y. Choquet-Bruhat, Class. Quant. Grav. 61781 (1989).

[16] A. R. Lugo and F. A. Schaposnik, Phys. Lett. B 467 (1999) 43.

[17] A. R. Lugo, E. F. Moreno and F. A. Schaposnik, Phys. Lett. B 473 (2000) 35.

[18] B. Hartmann, B.Kleihaus, J. Kunz, Mod.Phys.Lett.A15 (2000) 1003.

[19] Y. Brihaye and J. Kunz, Phys. Rev. D 50 (1994) 4175.

[20] W. Schönauer and R. Weiß, J. Comput. Appl. Math. 27 (1989) 279;

M. Schauder, R. Weiß and W. Schönauer, The CADSOL Program Package, Universität Karlsruhe, Interner Bericht Nr. 46/92 (1992).

[21] E. Radu, Phys. Rev. D 65 (2002) 044005.

[22] Y. Hosotani, J. Math. Phys. 43 (2002) 597.

[23] P. S. Jang, S. Y. Park and K. C. Wali, Phys. Rev. D 17 (1978) 1641.

[24] B. Rüber, Thesis, University of Bonn (1985).

[25] B. Kleihaus and J. Kunz, Phys. Rev. D 61 (2000) 025003. 


\section{Figure Captions}

Figure 1:

Typical spherically symmetric dyon solutions for the same value of Higgs self-coupling constant $\lambda=1$ and different values of $\Lambda, \alpha$. The total energy $E$ (in units $4 \pi \eta$ ) and the electric charge $Q_{e}$ are also marked.

\section{Figure 2:}

The energy per topological charge $E / n$ in units $4 \pi \eta$ (Figure 2a) and the electric charge per topological charge $Q_{e} / n$ (Figure $2 \mathrm{~b}$ ) are plotted as a function of the cosmological constant for dyon solutions with $\alpha=0.2$ and $\lambda=0.5$.

Figure 3:

The energy density $-T_{t}^{t}$ (Figure 3a), surfaces of constant energy density (Figure 3b), and $T_{\varphi}^{t}$ component of the energy-momentum tensor (in units of $4 \pi \eta$ ) (Figure 3c) for a dyon solution with topological charge $n=2$, total energy $E=5.522$, electric charge $Q_{e}=0.086$ and a vanishing Higgs self-coupling, as a function of the dimensionless compactified coordinates $\rho=\bar{r} \sin \theta$ and $z=\bar{r} \cos \theta$. Here $\bar{x}=r /(1+r)$ is the coordinate used in the numerical calculations. In Figure 3b, the inner surface correspond to a value of the energy density $\epsilon=4$, while the value for the outer surface is 9.5. The cosmological constant is $\Lambda=-6$.

Figure 4:

The $T_{\varphi}^{t}$ component of the energy-momentum tensor (in units $4 \pi \eta$ ) at $\theta=\pi / 4$ for dyon solutions with $\alpha=0.2, \lambda=0.5$, is shown for several values of the cosmological constant. The topological charge of these solutions is $n=3$. 


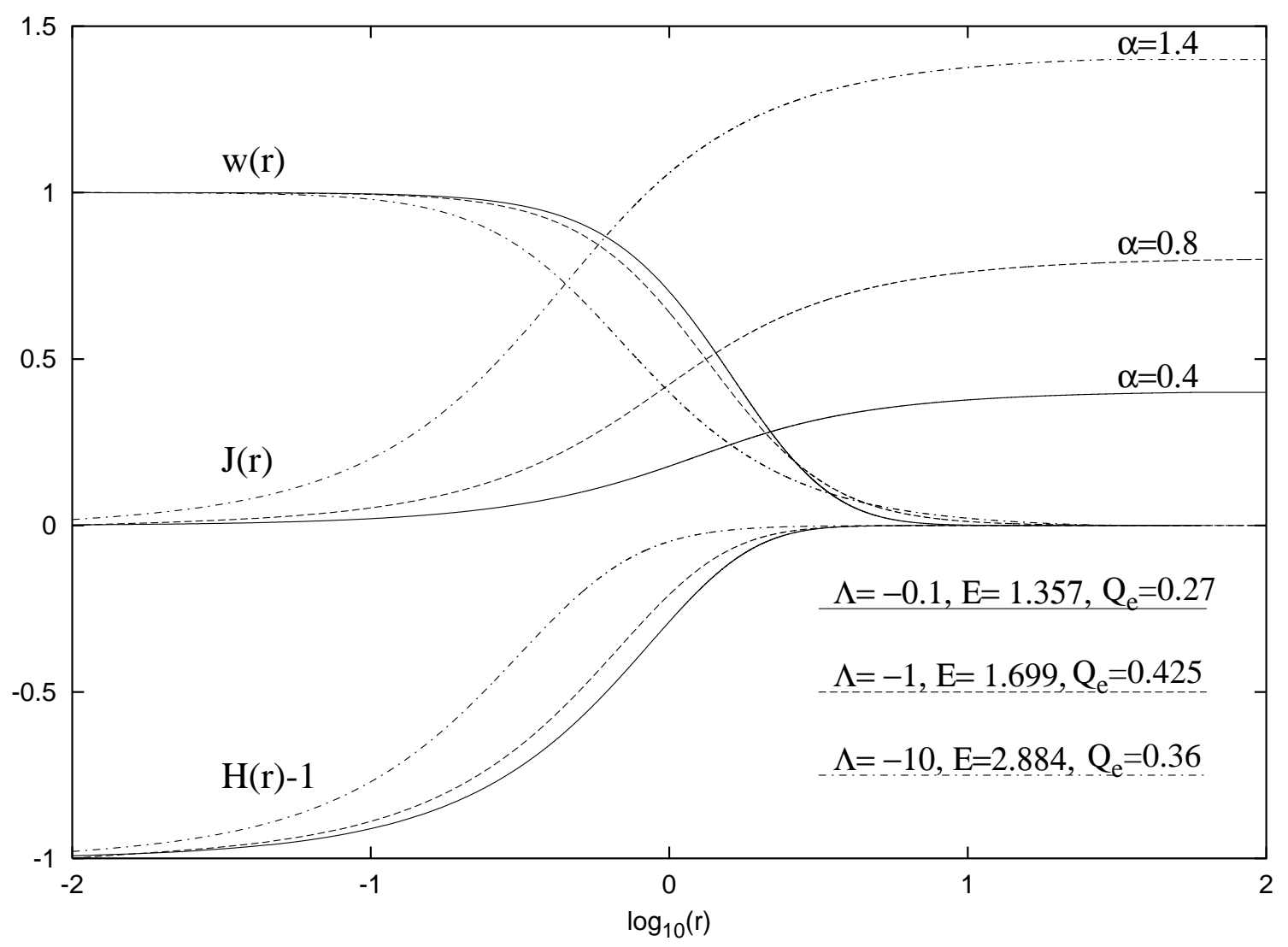

Figure 1. 


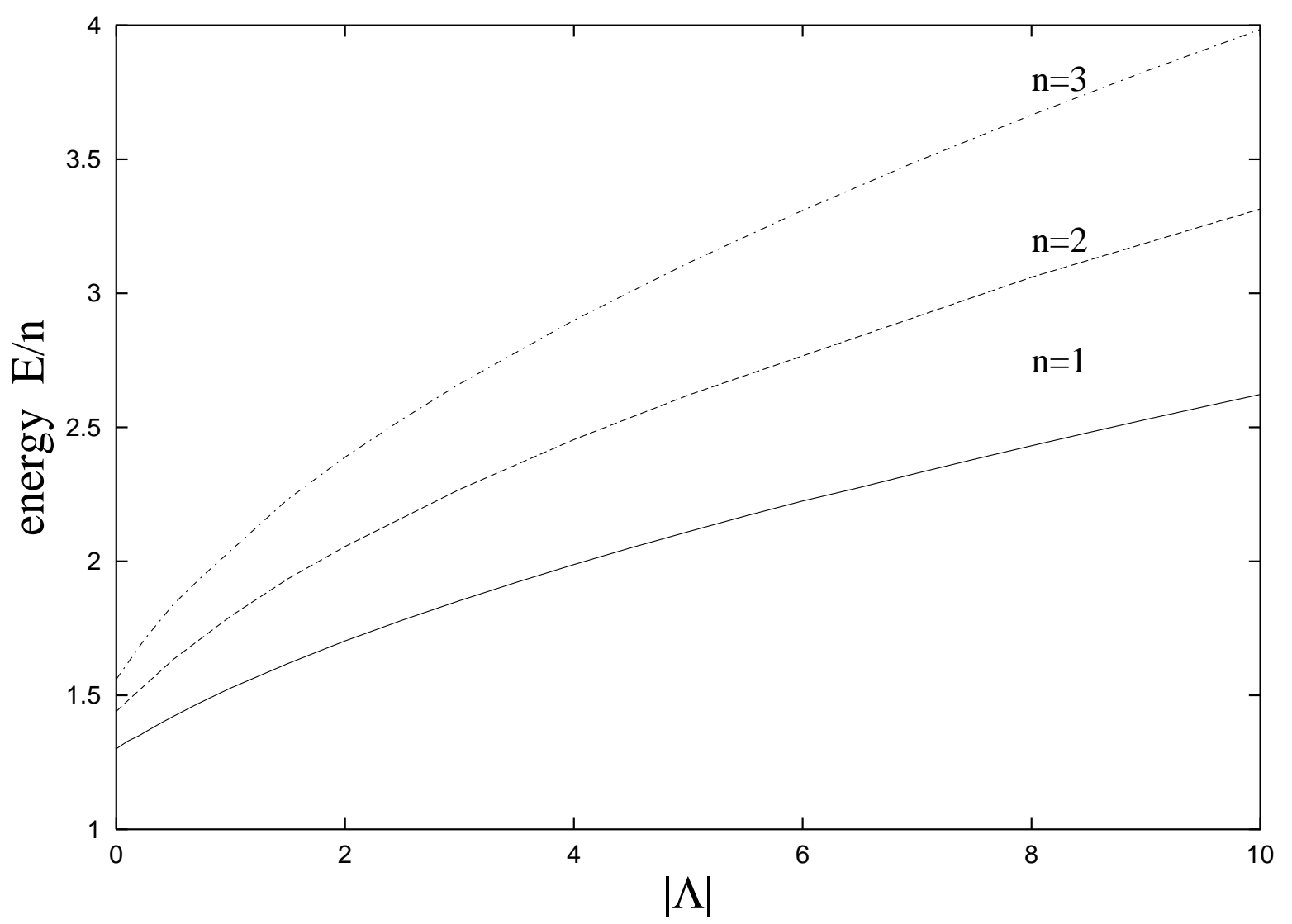

Figure 2a. 


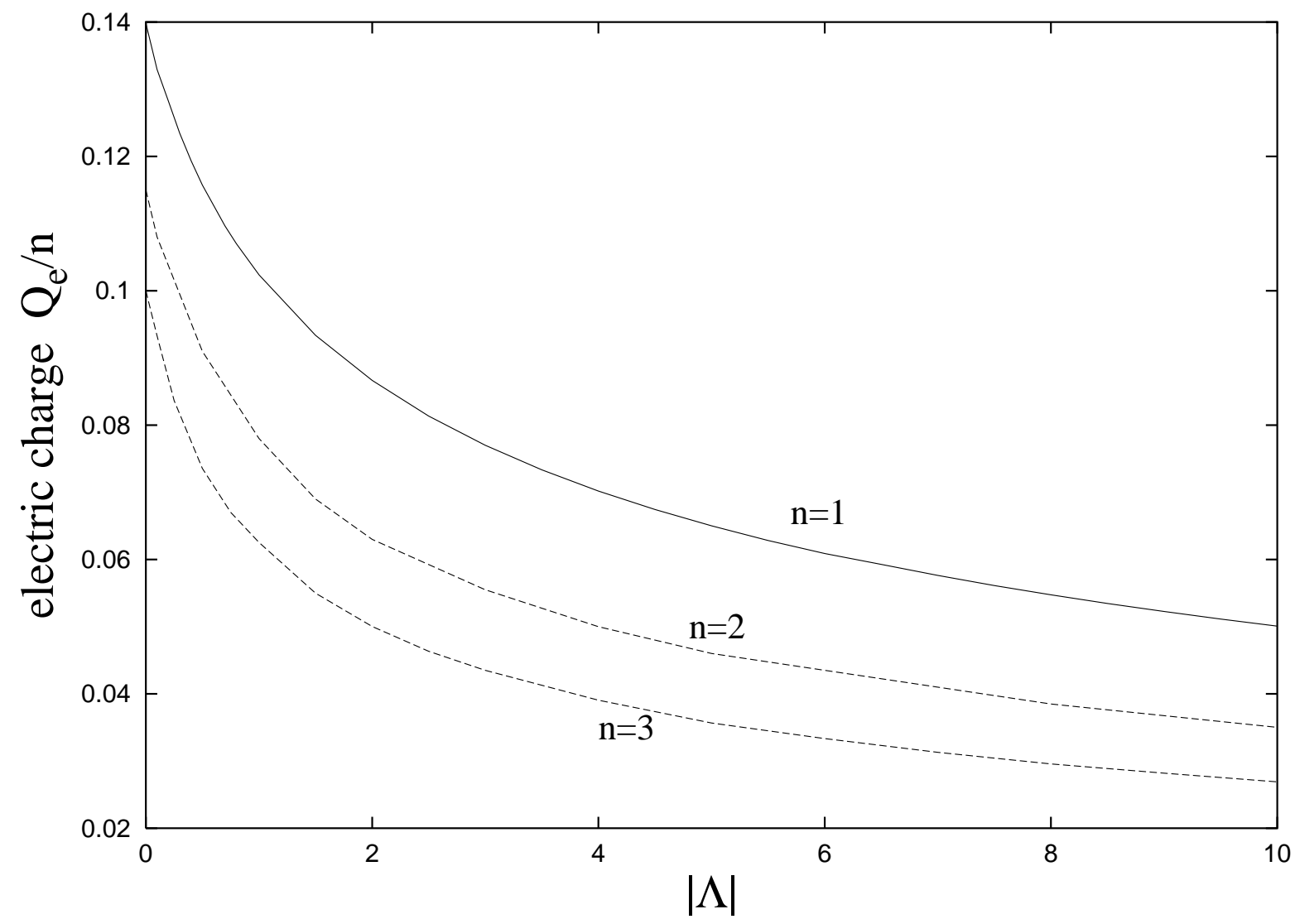

Figure 2b. 


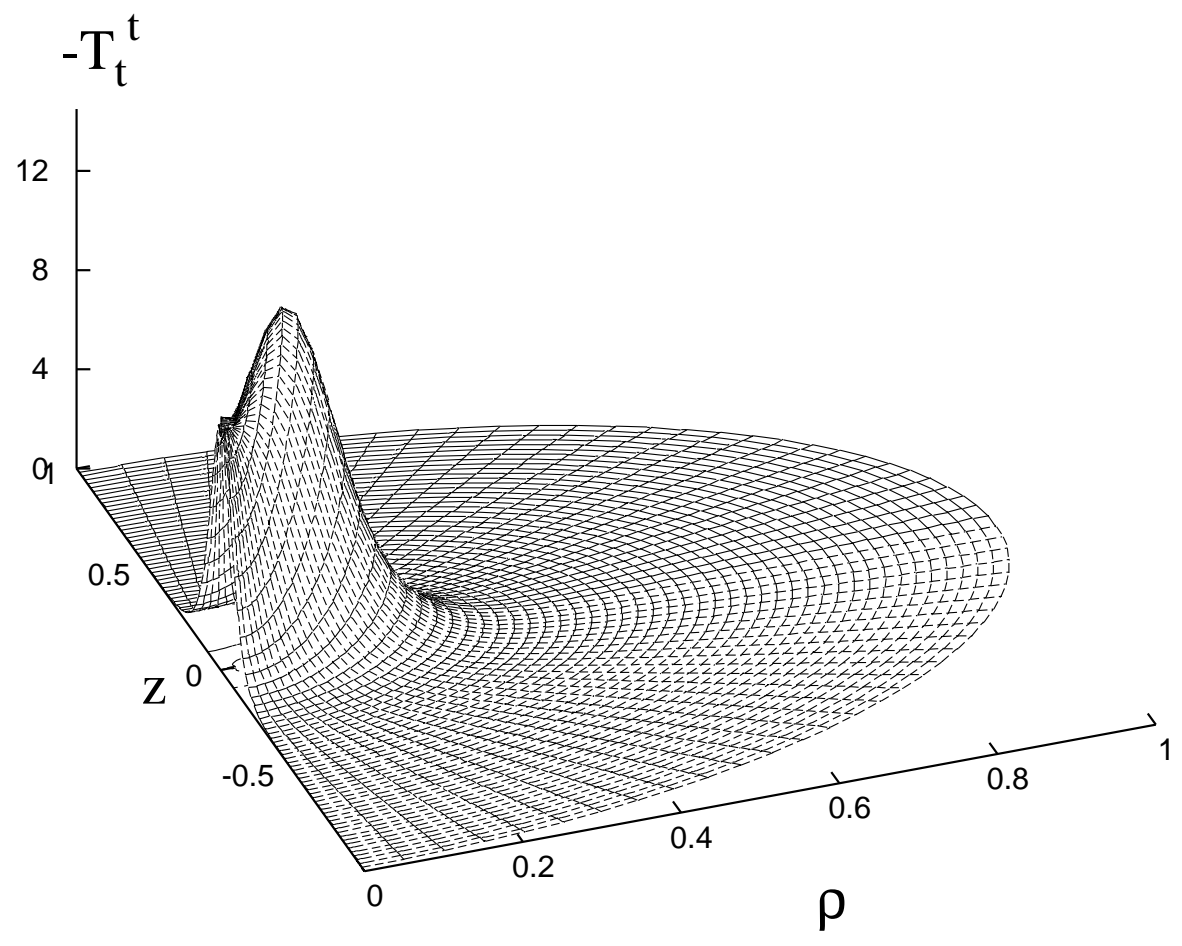

Figure 3a. 


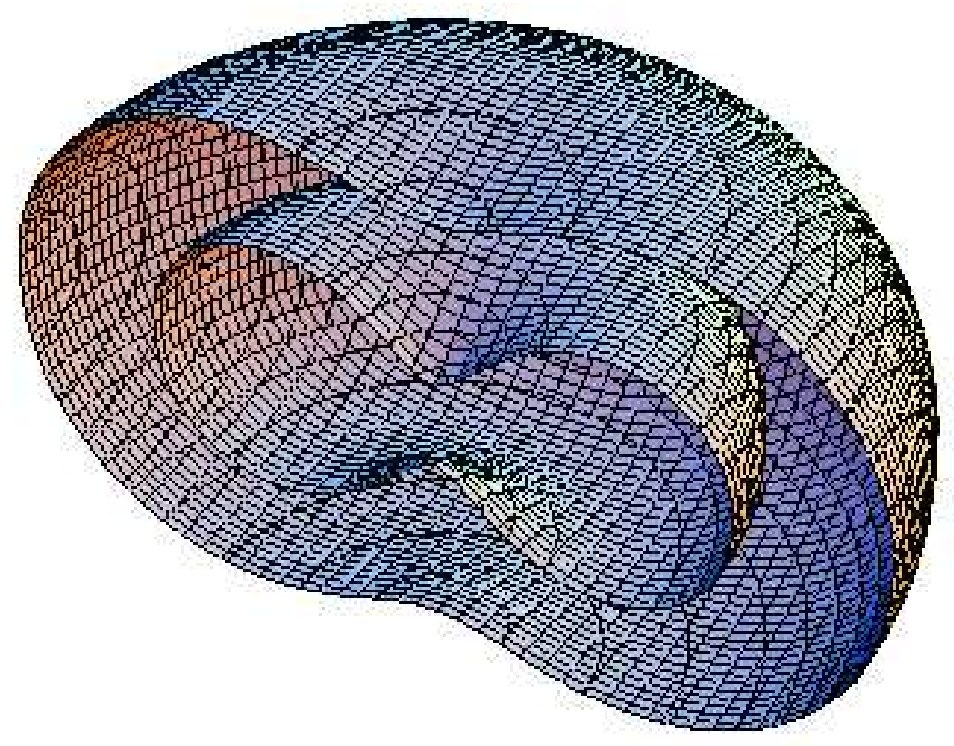

Figure 3b. 


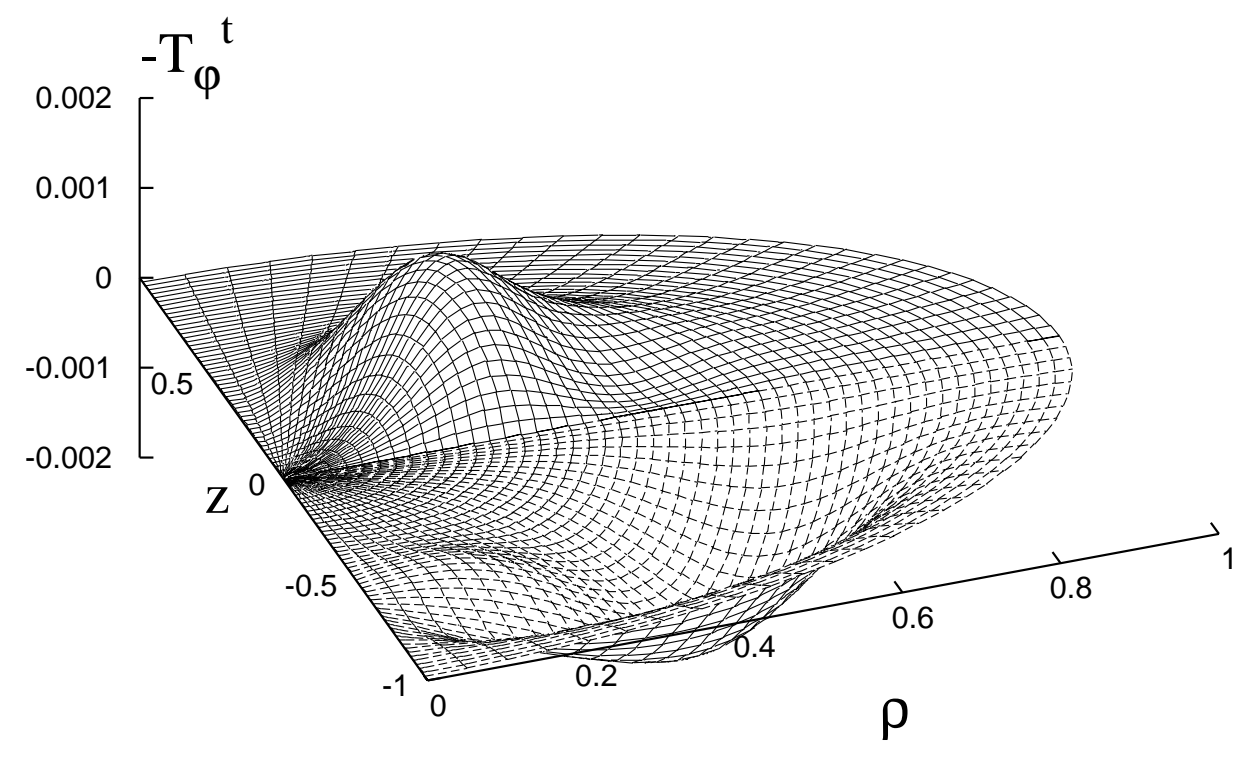

Figure 3c. 


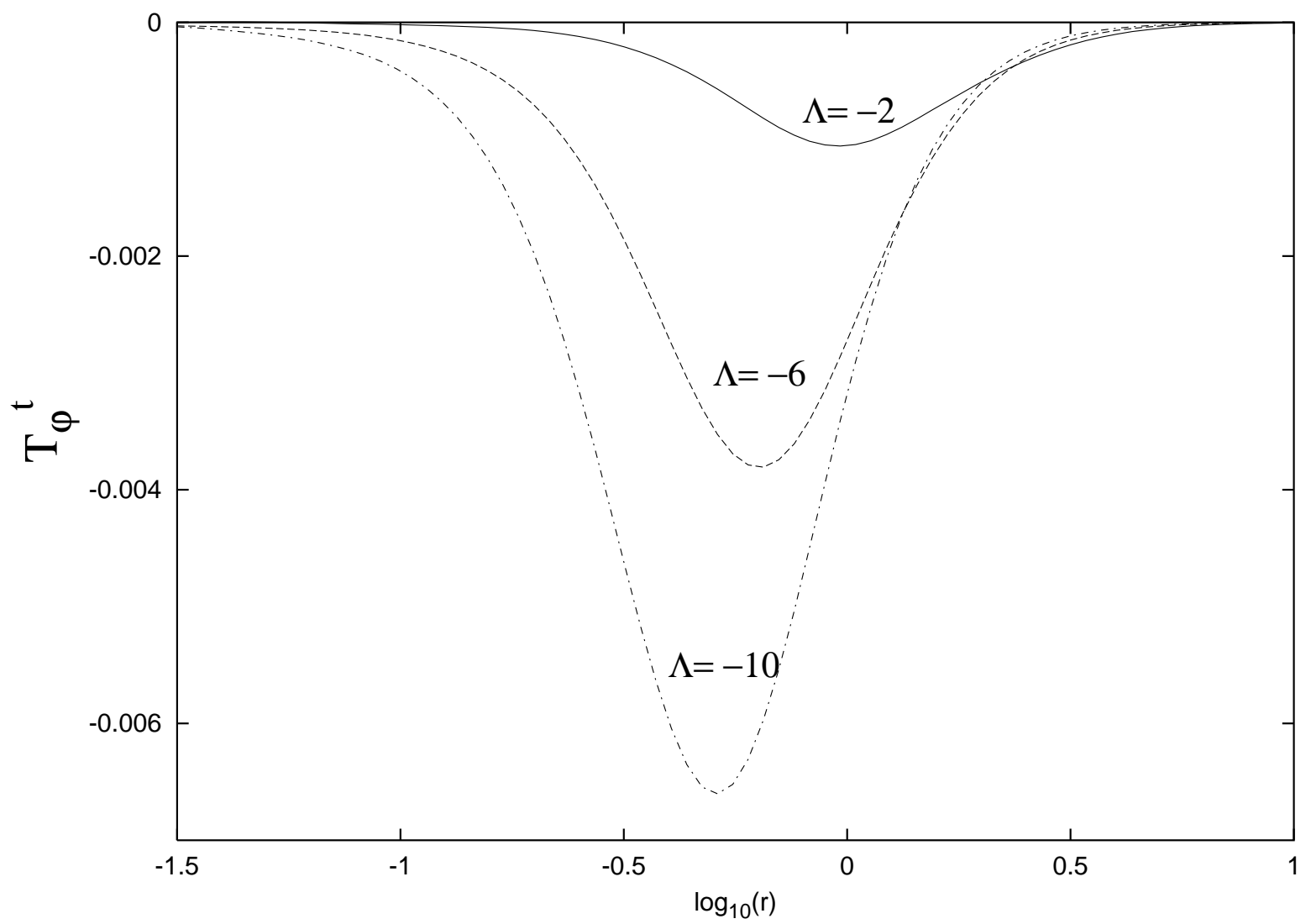

Figure 4. 